\title{
On Friedrichs-type inequalities in domains rarely perforated along the boundary
}

Yulia Koroleva ${ }^{1,2}$, Lars-Erik Persson ${ }^{2,3}$ and Peter Wall ${ }^{2^{*}}$

\footnotetext{
* Correspondence: peter.wall@Itu.se ${ }^{2}$ Department of Engineering Sciences and Mathematics, Luleå University of Technology, SE-971 87 Luleå, Sweden

Full list of author information is available at the end of the article
}

\begin{abstract}
This article is devoted to the Friedrichs inequality, where the domain is periodically perforated along the boundary. It is assumed that the functions satisfy homogeneous Neumann boundary conditions on the outer boundary and that they vanish on the perforation. In particular, it is proved that the best constant in the inequality converges to the best constant in a Friedrichs-type inequality as the size of the perforation goes to zero much faster than the period of perforation. The limit Friedrichs-type inequality is valid for functions in the Sobolev space $H^{1}$.

AMS 2010 Subject Classification: 39A10; 39A11; 39A70; 39B62; 41A44; 45A05.
\end{abstract}

Keywords: Friedrichs-type inequalities, homogenization, perforated along the boundary

\section{Introduction}

This article deals with Friedrichs-type inequalities for functions defined on domains which have a periodic perforation along the boundary. The size, shape and distribution of the perforation are described by a small parameter. It is assumed that the perforation is "rare", i.e., the size of the local perforation is much smaller than the period of perforation. Moreover, we consider the case where the functions satisfy a homogeneous Neumann condition on the part of the boundary corresponding to the domain without perforation and vanish on the perforation. The questions we are interested in are; how does the best constant in the Friedrichs-type inequality depend on the small parameter and what happens in the limit case where the parameter tends to zero? In particular, we will prove that the best constant converges to the best constant in a different type of Friedrichs inequality. The limit inequality is valid for all functions in the Sobolev space $H^{1}$.

Similar questions, with different types of microheterogeneities in a neighborhood of the boundary, were studied in [1-5]. In [1] (see also [2]), domains with a periodical perforation along the boundary were considered and the precise asymptotics of the best constant in a Friedrichs-type inequality was established. It was assumed that the size of perforation and the period were of the same order. Two different cases with non-periodical perforation were considered in [4,5]. The convergence of the constant, as the size of perforation tends to zero, to the constant in the limit inequality was proved. In [3], a Friedrichs-type inequality was proved for functions vanishing on small periodically alternating pieces of the boundary. The length of the pieces where the

(C) 2011 Koroleva et al; licensee Springer. This is an Open Access article distributed under the terms of the Creative Commons Attribution License (http://creativecommons.org/licenses/by/2.0), which permits unrestricted use, distribution, and reproduction in any medium, provided the original work is properly cited. 
functions vanish was assumed to be of the same order as the length of the period. In particular, the precise asymptotics of the best constant, with respect to the small parameter describing the heterogeneous boundary condition, was derived.

\section{Preliminaries and statement of the problem}

Let $\Omega \subset \mathbb{R}^{2}$ be a bounded domain such that the boundary, $\partial \Omega$, is Lipschitz continuous. Suppose that coordinates $\left(x_{1}, x_{2}\right)$ are used in $\Omega$. Introduce local coordinates $(s, t)$ in a small neighborhood of $\partial \Omega$ in the following way: choose the origin $O \in \partial \Omega$ and a point $P(s, t)$ in a neighborhood of $\partial \Omega$. Then, $t$ is the distance from $P$ to the boundary and $s$ is the counter clock-wise length of the boundary from $O$ to the point $P_{1}(s, t)$, where $P_{1}$ is the point for which $t=\left|\overline{P P}_{1}\right|$ (see Figure 1).

Consider a semi-strip $B=\left\{\xi \in \mathbb{R}^{2}: 0<\xi_{1}<1, \xi_{2}>0\right\}$ and a closed set $T_{\mu} \subset B$ depending on a small parameter $\mu \in(0,1]$ which characterizes the size and the shape of the perforation (see Figure 2).

We study the case when $T_{\mu}$ is shrinking in a uniform way as $\mu$ goes to zero. Moreover, we assume that $T_{\mu}$ is uniformly bounded with respect to $\mu$, i.e., there exists $\rho \in$ $\mathbb{R}, \rho>0$ such that $T_{\mu} \subset\left\{\xi \in \mathbb{R}^{2}: 0<\xi_{1}<1,0<\xi_{2}<\rho\right\}$ for all $\mu \in(0,1]$.

Let $T_{\mu}^{1}$ be 1-periodic extension of $T_{\mu}$ with respect to $\xi_{1}$ and $T_{\mu}^{\varepsilon}$ is the image of $T_{\mu}^{1}$ under the mapping $s=\varepsilon \xi_{1}, t=\varepsilon \xi_{2}$, where $\varepsilon$ is a small parameter, $0<\varepsilon \ll 1, \frac{1}{\varepsilon} \in \mathrm{N}$. Define the domain $\Omega_{\varepsilon}=\Omega \backslash T_{\mu}^{\varepsilon}$ (see Figure 2). Further, we assume that $\mu=\mu(\varepsilon)$ and that

$$
\mu=\mu(\varepsilon) \rightarrow 0 \quad \text { as } \varepsilon \rightarrow 0 .
$$

Hence, $\varepsilon>0$ is a parameter which describes both the size of the perforation and the length of the period.

Consider the following spectral problem:

$$
\begin{cases}-\Delta u_{\varepsilon}=\lambda_{\varepsilon} u_{\varepsilon} & \text { in } \Omega_{\varepsilon} \\ u_{\varepsilon}=0 & \text { on } T_{\mu^{\prime}}^{\varepsilon} \\ \frac{\partial u_{\varepsilon}}{\partial v}=0 & \text { on } \partial \Omega .\end{cases}
$$

Here $v$ denotes the unit outward normal to $\Omega$. The limit problem for (2) depends on how fast the size of the perforation goes to zero relative the length of the period. It was proved in [6] (see also [7]) that if the perforation is "rare", i.e., the size of the local perforation goes to zero much faster than the period of perforation, then the limit problem for (2) is the Robin boundary value problem

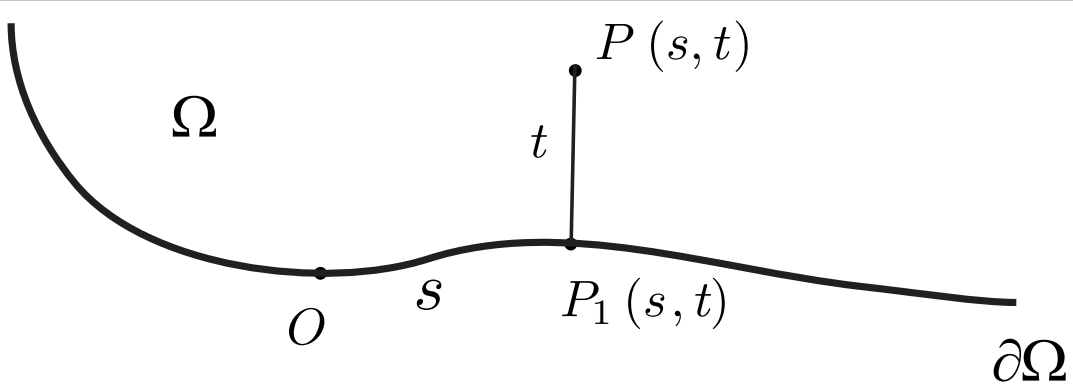

Figure 1 The local coordinate system 


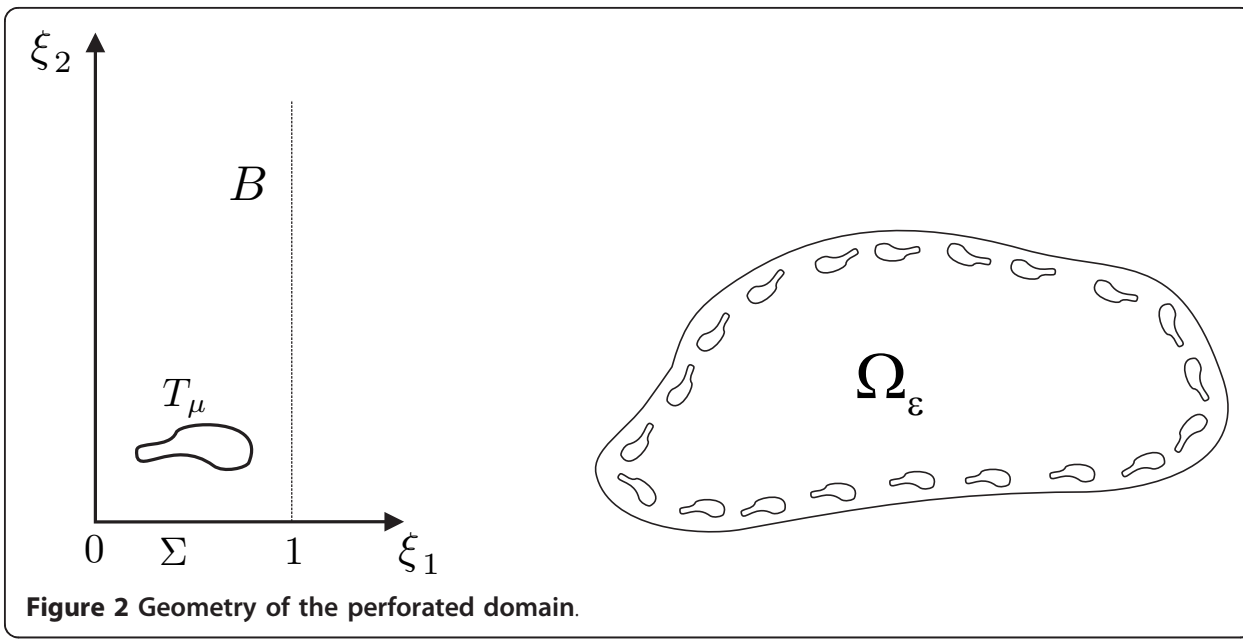

$$
\left\{\begin{array}{l}
-\Delta u_{0}=\lambda_{0} u_{0} \text { in } \Omega \\
\frac{\partial u_{0}}{\partial v}+p u_{0}=0 \text { on } \partial \Omega
\end{array}\right.
$$

where $0<p<\infty$. The precise meaning of that the perforation is rare is given in (14) later on. The faster the size of the local perforation goes to zero the smaller $p$ will be. There is a critical speed which gives that $p$ is equal to zero, see $[6,7]$. In such situations the limit problem is of Neumann type. The limit problem is of Dirichlet type $(p=\infty)$ when the size of the local perforation does not go to zero "fast enough" relative the period of perforation.

According to the general theory of elliptic operators, there exist countable sets $\left\{\lambda_{\varepsilon}^{k}\right\}$ and $\left\{\lambda_{0}^{k}\right\}$ of eigenvalues of (2) and (3) which satisfy

$$
0<\lambda_{\varepsilon}^{1} \leq \lambda_{\varepsilon}^{2} \leq \cdots \leq \lambda_{\varepsilon}^{k} \leq \cdots, \quad 0<\lambda_{0}^{1} \leq \lambda_{0}^{2} \leq \cdots \leq \lambda_{0}^{k} \leq \cdots .
$$

Using the same arguments as in [4], it follows that $\lambda_{\varepsilon}^{1}>0$. This together with the variational formulation of the smallest eigenvalue of (2) lead to the following Friedrichs-type inequality for functions $u \in H^{1}(\Omega)$ which vanish on $T_{\mu}^{\varepsilon}$ :

$$
\int_{\Omega_{\varepsilon}} u^{2} d x \leq K_{\varepsilon} \int_{\Omega_{\varepsilon}}|\nabla u|^{2} d x
$$

where $K_{\varepsilon}$ is the best constant and is given by

$$
K_{\varepsilon}=\frac{1}{\lambda_{\varepsilon}^{1}} .
$$

In the case with $p=\infty$ (Dirichlet boundary conditions in the limit problem) the smallest eigenvalue $\lambda_{0}^{1}$ for the limit problem is related to the best constant in the Friedrichs inequality for functions in $H_{0}^{1}(\Omega)$. Indeed, via the variational formulation of $\lambda_{0}^{1}$ we have that

$$
\int_{\Omega} u^{2} d x \leq K_{0} \int_{\Omega}|\nabla u|^{2} d x,
$$

where the best constant is given by $K_{0}=1 / \lambda_{0}^{1}$. 
A geometrical proof of that $K_{\varepsilon} \rightarrow K_{0}$ was presented in [4] for the case $p=\infty$. The goal of this article is to answer the following questions, in the case $0<p<\infty$ : (1) Is there a Friedrichs-type inequality related to the limit Robin boundary value problem? (2) If the answer on the first questions is yes, how is then $K_{\varepsilon}$ related to the best constant, $K_{0}$, in this Friedrichs-type inequality. We will see that there is such a Friedrichstype inequality and we present a result describing the asymptotic relation between $K_{\varepsilon}$ and $K_{0}$. Moreover, as a result of our analysis we also obtain the convergence of the eigenvalues, $\lambda_{\varepsilon}^{k} \rightarrow \lambda_{0}^{k}$, for the case $0<p<\infty$.

\section{The main results}

The following Friedrichs-type inequality holds for functions in $H^{1}(\Omega)$ :

Proposition 1 There exists a constant $K_{0}>0$ such that

$$
\int_{\Omega} u^{2} d x \leq K_{0}\left(\int_{\Omega}|\nabla u|^{2} d x+p \int_{\partial \Omega} u^{2} d s\right)
$$

for any $u \in H^{1}(\Omega)$. Moreover, the best constant is $K_{0}=1 / \lambda_{0}^{1}$, where $\lambda_{0}^{1}$ is the smallest eigenvalue in the limit problem (3).

Proof. The variational formulation of the smallest eigenvalue of the limit problem (3) is

$$
\lambda_{0}^{1}=\min _{u \in H^{1}(\Omega) \backslash\{0\}}\left\{\frac{\int_{\Omega}|\nabla u|^{2} d x+p \int_{\partial \Omega} u^{2} d s}{\int_{\Omega} u^{2} d x}\right\} .
$$

For details, see paragraph 2.5 in [8]. From this, it is clear that the inequality (7) holds. It also follows that the best constant is $1 / \lambda_{0}^{1}$.

Let us define the following set of functions:

$$
W=\left\{u \in H^{1}(\Omega): \frac{\partial u}{\partial v}+p u=0 \quad \text { on } \partial \Omega, 0<p<\infty\right\} .
$$

Note that solutions of the limit problem (3) belong to $W$. We remark that an inequality of the form (6) cannot be valid for functions in $W$. Indeed,

Proposition 2 There is no $C>0$ such that the inequality

$$
\int_{\Omega} u^{2} d x \leq C \int_{\Omega}|\nabla u|^{2} d x
$$

holds for all functions in W.

Proof. We prove the statement by a counter example. Let

$$
K_{m}=\{x \in \Omega: \operatorname{dist}(x, \partial \Omega) \geq 1 / m, \quad m \in \mathbb{N}\} .
$$

Define the function $u_{m}$ such that $u_{m}=m+p$ on $K_{m}$ and $u_{m}=m$ on $\partial \Omega$. It is possible to construct a smooth transition from $K_{m}$ to $\partial \Omega$ such that $\partial u_{m} / \partial v+p u_{m}=0$ on $\partial \Omega$ and

$$
\left|\nabla u_{m}\right|^{2} \leq k\left(\frac{m+p-m}{1 / m}\right)^{2}=k p^{2} m^{2}
$$


in $\Omega \backslash K_{m}$ for some constant $k$. Note that $\nabla u_{m}=0$ on $K_{m}$ and that $\left|\Omega \backslash K_{m}\right| \sim 1 / m(\sim$ means asymptotically equal to). We get that

$$
\frac{\int_{\Omega}\left|\nabla u_{m}\right|^{2} d x}{\int_{\Omega} u_{m}^{2} d x} \leq \frac{k p^{2} m^{2}\left|\Omega \backslash K_{m}\right|}{\int_{\Omega} u_{m}^{2} d x} \leq \frac{k p^{2} m^{2}\left|\Omega \backslash K_{m}\right|}{m^{2}|\Omega|} \rightarrow 0
$$

as $m \rightarrow \infty$. Thus, (8) cannot hold.

We will now consider how to estimate the difference between the best constants, $K_{0}$ and $K_{\varepsilon}$, in the inequalities (7) and (4). First, observe that by Proposition 1 and (5) we have that

$$
\left|K_{\varepsilon}-K_{0}\right|=\left|\frac{1}{\lambda_{\varepsilon}^{1}}-\frac{1}{\lambda_{0}^{1}}\right| .
$$

To estimate $\left|1 / \lambda_{\varepsilon}^{1}-1 / \lambda_{0}^{1}\right|$ we will use the ideas in the general method for estimating the difference between eigenvalues and eigenvectors of two operators defined on different spaces, which was introduced by Oleinik et al. [9], see also [10].

For the readers convenience, we review the main ideas in the method mentioned above. Indeed, let $H_{\varepsilon}$ and $H_{0}$ be separable Hilbert spaces with the inner products $\left(u_{\varepsilon}, v_{\varepsilon}\right)_{H_{\varepsilon}},(u, v)_{H_{0}}$ and norms $\left\|u_{\varepsilon}\right\|_{H_{\varepsilon}},\|u\|_{H_{0}}$, respectively; assume that $A_{\varepsilon} \in L\left(H_{\varepsilon}\right)$ and $A_{0} \in L\left(H_{0}\right)$ are linear continuous operators and $\operatorname{Im} A_{0} \subseteq V \subseteq H_{0}$, where $V$ is a linear subspace of $H_{0}$. The following conditions are supposed to hold:

C1 There exist linear continuous operators $R_{\varepsilon}: H_{\varepsilon} \rightarrow H_{O}$ and a constant $c>0$ such that

$$
\left(R_{\varepsilon} f, R_{\varepsilon} f\right)_{H_{\varepsilon}} \rightarrow c(f, f)_{H_{0}} \text { as } \varepsilon \rightarrow 0
$$

for any $f \in V$.

C2 The operators $A_{\varepsilon}: H_{\varepsilon} \rightarrow H_{\varepsilon}$ and $A_{0}: H_{0} \rightarrow H_{0}$ are positive, compact and selfadjoint. Moreover, $\sup _{\varepsilon}\left\|A_{\varepsilon}\right\|_{L\left(H_{\varepsilon}\right)}<+\infty$.

C3 For all $f \in V$ it holds that $\left\|A_{\varepsilon} R_{\varepsilon} f-R_{\varepsilon} A_{0} f\right\|_{H_{\varepsilon}} \rightarrow 0$ as $\varepsilon \rightarrow 0$.

C4 The sequence of operators $A_{\varepsilon}$ is uniformly compact in the following sense: if we take a sequence $\left\{f_{\varepsilon}\right\}$, where $f_{\varepsilon} \in H_{\varepsilon}$, such that $\sup _{\varepsilon}|| f_{\varepsilon} \|_{H_{\varepsilon}}<+\infty$, then there exist a subsequence $\left\{f_{\varepsilon_{k}}\right\}$ and vector $w_{0} \in V$ such that $\left\|A_{\varepsilon_{k}} f_{\varepsilon_{k}}-R_{\varepsilon_{k}} w_{0}\right\|_{H_{\varepsilon_{k}}} \rightarrow 0$ as $\varepsilon_{k} \rightarrow 0$.

Let us also introduce the spectral problems for operators $A_{\varepsilon}, A_{0}$ :

$$
\begin{aligned}
& A_{\varepsilon} u_{\varepsilon}^{k}=\mu_{\varepsilon}^{k} u_{\varepsilon^{\prime}}^{k} \quad \mu_{\varepsilon}^{1} \geq \mu_{\varepsilon}^{2} \geq \cdots, \quad k=1,2, \ldots\left(u_{\varepsilon}^{l}, u_{\varepsilon}^{m}\right)=\delta_{l m}, \\
& A_{0} u_{0}^{k}=\mu_{0}^{k} u_{0}^{k}, \quad \mu_{0}^{1} \geq \mu_{0}^{2} \geq \cdots, \quad k=1,2, \ldots\left(u_{0}^{l}, u_{0}^{m}\right)=\delta_{l m},
\end{aligned}
$$

where $\delta_{l m}$ is the Kronecker symbol, the eigenvalues $\mu_{\varepsilon^{\prime}}^{k} \mu_{0}^{k}$ are repeated according to their multiplicities. The following lemma holds true (see [9, Chapter III]).

Lemma 3 Suppose that the conditions C1-C4 are valid. Then, there is a sequence $\left\{\beta_{\varepsilon}^{k}\right\}$ such that $\beta_{\varepsilon}^{k} \rightarrow 0$ as $\varepsilon \rightarrow 0,0<\beta_{\varepsilon}^{k}<\mu_{0}^{k}$ and the following estimate:

$$
\left|\mu_{\varepsilon}^{k}-\mu_{0}^{k}\right| \leq \frac{\mu_{0}^{k} c^{-\frac{1}{2}}}{\mu_{0}^{k}-\beta_{\varepsilon}^{k}} \sup _{v \in N\left(\mu_{0}^{k}, A_{0}\right),\|v\|_{H_{0}=1}}\left\|A_{\varepsilon} R_{\varepsilon} v-R_{\varepsilon} A_{0} v\right\|_{H_{\varepsilon}}
$$


holds, where $N\left(\mu_{0}^{k}, A_{0}\right)=\left\{v \in H_{0}: A_{0} v=\mu_{0}^{k} v\right\}$.

Let us now give a more precise definition of that the perforation is rare. Indeed, introduce the space $V_{\mu(\varepsilon)}$ as the closure of the set of functions in $v \in C^{\infty}\left(\mathbb{R}^{2} \cap\left\{\xi_{2}>\right.\right.$ $0\})$ which are 1-periodic with respect to $\xi_{1}$, vanishing on $T_{\mu(\varepsilon)}$ and with finite $\int_{B}|\nabla v|^{2} d \xi$. The closure is with respect to the norm $\|v\|=\int_{B}|\nabla v|^{2} d \xi+\int_{\sum} v^{2} d \xi_{1}$. Moreover, define the value

$$
\eta_{\mu(\varepsilon)}=\inf _{v \in V_{\mu(\varepsilon)} \backslash\{0\}} \frac{\int_{B}|\nabla v|^{2} d \xi}{\int_{\Sigma} v^{2} d \xi_{1}},
$$

where $\Sigma:=\partial B \cap\left\{\xi_{2}=0\right\}$ (see Figure 2). Moreover, we define the number $p$ as

$$
\lim _{\varepsilon \rightarrow 0} \frac{\eta_{\mu(\varepsilon)}}{\varepsilon}=p .
$$

In fact, the number $p$ corresponds to the ratio between measure of small set $T_{\mu}$ and the length of period, i.e., it describes how much of the Dirichlet condition per cell of periodicity we have.

We will now prove the following estimate for $\left|K_{\varepsilon}-K_{0}\right|$ :

Theorem 4 Let $K_{\varepsilon}$ and $K_{0}$ be the constants in (4) and (7). If $0<p<\infty$ is defined by (14), then there exists a constant $C$, independent of $\varepsilon$, such that

$$
\left|K_{\varepsilon}-K_{0}\right| \leq C\left(\sqrt{\eta_{\mu(\varepsilon)}}+\left|\frac{\eta_{\mu(\varepsilon)}}{\varepsilon}-p\right|+\sqrt{\varepsilon \eta_{\mu(\varepsilon)}}\right) .
$$

Proof. By (9) we will have an estimate of $\left|K_{\varepsilon}-K_{0}\right|$ if we have an estimate of $\left|1 / \lambda_{\varepsilon}^{1}-1 / \lambda_{0}^{1}\right|$. In order to obtain such an estimate we will use the result in Lemma 3.

Indeed, we introduce two auxiliary problems:

$$
\begin{cases}-\Delta u_{\varepsilon}=f & \text { in } \Omega_{\varepsilon} \\ u_{\varepsilon}=0 & \text { on } \mathrm{T}_{\mu^{\prime}}^{\varepsilon} \\ \frac{\partial u_{\varepsilon}}{\partial v}=0 & \text { on } \partial \Omega\end{cases}
$$

and the corresponding limit problem

$$
\left\{\begin{array}{l}
-\Delta u_{0}=f \quad \text { in } \Omega, \\
\frac{\partial u_{0}}{\partial v}+p u_{0}=0 \text { on } \partial \Omega,
\end{array}\right.
$$

where $f \in L^{2}(\Omega)$ and $p$ satisfies (14). The fact that (17) is the limit problem for (16) for any $f$ was established in [6]. More precisely, it was proved that if $u_{\varepsilon} \in H^{1}\left(\Omega_{\varepsilon}\right)$ and $u_{0} \in H^{1}(\Omega)$ are weak solutions of (16) and (17), then $u_{\varepsilon} \rightarrow u_{0}$ weakly in $H^{1}(\Omega)$ as $\varepsilon \rightarrow$ 0 which implies the convergence

$$
\left\|u_{\varepsilon}-u_{0}\right\|_{L^{2}(\Omega)} \rightarrow 0 \quad \text { as } \varepsilon \rightarrow 0 .
$$

Note that here and from now on, $u_{\varepsilon}$ is assumed to be defined in whole $\Omega$ and vanishing on $T_{\mu}^{\varepsilon}$.

Let us now prove the following estimates for the solutions of (16) and (17):

$$
\left\|u_{\varepsilon}\right\|_{H^{1}\left(\Omega_{\varepsilon}\right)} \leq k_{1}\|f\|_{L^{2}\left(\Omega_{\varepsilon}\right)}
$$


and

$$
\left\|u_{0}\right\|_{H^{1}(\Omega)} \leq k_{2}\|f\|_{L^{2}(\Omega)}
$$

where $k_{1}$ and $k_{2}$ are independent of $\varepsilon$. First, we recall that using the technique developed in [4] (see also [5]), one can prove the following Friedrichs-type inequality for functions $w$ belonging to $H^{1}(\Omega)$ and vanishing on $T_{\mu}^{\varepsilon}$ :

$$
\int_{\Omega} w^{2} d x \leq K \int_{\Omega}|\nabla w|^{2} d x
$$

where $K$ does not depend on $\varepsilon$. In particular, the inequality (21) implies that the solution of (16) satisfies the estimate

$$
\int_{\Omega_{\varepsilon}} u_{\varepsilon}^{2} d x \leq K \int_{\Omega_{\varepsilon}}\left|\nabla u_{\varepsilon}\right|^{2} d x
$$

By choosing $u_{\varepsilon}$ as the test function in the weak formulation of (16), we have

$$
\int_{\Omega_{\varepsilon}}\left|\nabla u_{\varepsilon}\right|^{2} d x=\int_{\Omega_{\varepsilon}} f u_{\varepsilon} d x .
$$

Using the Hölder inequality and (22), we obtain that

$$
\left\|\nabla u_{\varepsilon}\right\|_{L^{2}\left(\Omega_{\varepsilon}\right)} \leq \sqrt{K}\|f\|_{L^{2}\left(\Omega_{\varepsilon}\right)} .
$$

From this and (22) the estimate (19) follows, with $k_{1}=\sqrt{K(1+K)}$. Let us now prove the estimate (20). Indeed, we start by showing that for any $w \in H^{1}(\Omega) \backslash\{0\}$ there exists a constant $M$ which does not depend on $w$ such that

$$
\int_{\Omega}|\nabla w|^{2} d x\left|p \int_{\partial \Omega} w^{2} d s \geq M\right| \mid w \|_{H^{1}(\Omega)}^{2} .
$$

Suppose that the contradiction holds: i.e., that for any $m$ there exists $w_{m} \in H^{1}(\Omega) \backslash\{0\}$ such that

$$
\int_{\Omega}\left|\nabla w_{m}\right|^{2} d x+p \int_{\partial \Omega} w_{m}^{2} d s<\frac{1}{m}\left\|w_{m}\right\|_{H^{1}(\Omega)}^{2} .
$$

Denote $v_{m}=w_{m} /\left\|w_{m}\right\|_{H^{1}(\Omega)}$. Then,

$$
\left\|v_{m}\right\|_{H^{1}(\Omega)}=1
$$

and

$$
\int_{\Omega}\left|\nabla v_{m}\right|^{2} d x+p \int_{\partial \Omega} v_{m}^{2} d s<\frac{1}{m}
$$

By the inequalities (7) and (25), we have that

$$
\int_{\Omega} v_{m}^{2} d x \leq K_{0}\left(\int_{\Omega}\left|\nabla v_{m}\right|^{2} d x+p \int_{\partial \Omega} v_{m}^{2} d s\right)<\frac{K_{0}}{m} .
$$


From (25) and (26), it follows that $v_{m} \rightarrow 0$ in $H^{1}(\Omega)$, which contradicts to (24). Thus, the estimate (23) is proved. Choosing $u_{0}$ as a test function in the weak formulation of (17) leads to the identity

$$
\int_{\Omega}\left|\nabla u_{0}\right|^{2} d x+p \int_{\partial \Omega} u_{0}^{2} d s=\int_{\Omega} f u_{0} d x .
$$

By applying (23) to $u_{0}$, using (27) and the Cauchy-Schwarz inequality, we get that

$$
\left\|u_{0}\right\|_{H^{1}(\Omega)} \leq \frac{1}{M}\|f\|_{L^{2}(\Omega)}
$$

which is estimate (20) with $k_{2}=1 / M$.

To estimate $\left|1 / \lambda_{\varepsilon}^{1}-1 / \lambda_{0}^{1}\right|$ we will now use the method, which was described above, for estimating the difference between eigenvalues. Indeed, define the spaces $H_{\varepsilon}=L^{2}$ $\left(\Omega_{\varepsilon}\right), H_{0}=V=L^{2}(\Omega)$ and the restriction operator $R_{\varepsilon}: H_{0} \rightarrow H_{\varepsilon}$. Define the operators $A_{\varepsilon}$ and $A_{0}$ in the following way: $A_{\varepsilon} f=u_{\varepsilon}$ and $A_{0} f=u_{0}$, where $u_{\varepsilon}$ and $u_{0}$ are the weak solutions of problems (16) and (17), respectively. Let us verify the conditions C1-C4.

The condition $\mathrm{C} 1$ is valid with $c=1$. Indeed, take $f \in V$. Then,

$$
\left(R_{\varepsilon} f, R_{\varepsilon} f\right)_{\varepsilon}=\int_{\Omega_{\varepsilon}} f^{2}(x) d x \rightarrow \int_{\Omega} f^{2}(x) d x=(f, f)_{0}
$$

as $\varepsilon \rightarrow 0$ due to the fact that measure of $T_{\mu}^{\varepsilon} \rightarrow 0$ as $\varepsilon \rightarrow 0$.

Let us verify the condition C2. First, we prove that the operator $A_{\varepsilon}$ is self-adjoint. Let $f$ and $g$ be functions in $L^{2}\left(\Omega_{\varepsilon}\right)$ and define $u_{\varepsilon}=A_{\varepsilon} f$ and $v_{\varepsilon}=A_{\varepsilon} g$. If we chose $v_{\varepsilon}$ as test function in the weak formulation of (16) with $f$ in the right-hand side and $u_{\varepsilon}$ as a test function in the case when the right-hand side is $g$, then we obtain that

$$
\int_{\Omega_{\varepsilon}} f v_{\varepsilon} d x=\int_{\Omega_{\varepsilon}} \nabla u_{\varepsilon} \cdot \nabla v_{\varepsilon} d x=\int_{\Omega_{\varepsilon}} g u_{\varepsilon} d x .
$$

Hence,

$$
\left(A_{\varepsilon} f, g\right)_{L^{2}\left(\Omega_{\varepsilon}\right)}=\left(u_{\varepsilon}, g\right)_{L^{2}\left(\Omega_{\varepsilon}\right)}=\int_{\Omega_{\varepsilon}} \nabla v_{\varepsilon} \cdot \nabla u_{\varepsilon} d x=\left(f, v_{\varepsilon}\right)_{L^{2}\left(\Omega_{\varepsilon}\right)}=\left(f, A_{\varepsilon} g\right)_{L^{2}\left(\Omega_{\varepsilon}\right)} .
$$

Now, we prove the operator $A_{0}$ is self-adjoint. Define $u_{0}=A_{0} f$ and $v_{0}=A_{0} g$, where $f$, $g \in L^{2}(\Omega)$. According to the weak formulation of (17), we find that

$$
\begin{gathered}
\left(A_{0} f, g\right)_{L^{2}(\Omega)}=\int_{\Omega} u_{0} g d x=\int_{\Omega} \nabla v_{0} \cdot \nabla u_{0} d x-\int_{\partial \Omega} u_{0} \frac{\partial v_{0}}{\partial v} d s \\
=\int_{\Omega} \nabla v_{0} \cdot \nabla u_{0} d x+p \int_{\partial \Omega} u_{0} v_{0} d s=\int_{\Omega} \nabla u_{0} \cdot \nabla v_{0} d x-\int_{\partial \Omega} v_{0} \frac{\partial u_{0}}{\partial v} d s \\
=\int_{\Omega} f v_{0} d x=\left(f, A_{0} g\right)_{L^{2}(\Omega)} .
\end{gathered}
$$


That the operator $A_{\varepsilon}$ is positive follows from

$$
\left(A_{\varepsilon} f, f\right)_{L^{2}(\Omega)}=\int_{\Omega} u_{\varepsilon} f d x=\int_{\Omega}\left|\nabla u_{\varepsilon}\right|^{2} d x>0
$$

if $u_{\varepsilon} \neq 0$ (i.e., $f \neq 0$ ). Similarly, we obtain that $A_{0}$ is positive. Indeed, the weak formulation of (17) gives

$$
\begin{aligned}
\left(A_{0} f, f\right)_{L^{2}(\Omega)} & =\int_{\Omega} u_{0} f d x=\int_{\Omega}\left|\nabla u_{0}\right|^{2} d x-\int_{\partial \Omega} u_{0} \frac{\partial u_{0}}{\partial v} d s \\
& =\int_{\Omega}\left|\nabla u_{0}\right|^{2} d x+p \int_{\partial \Omega} u_{0}^{2} d s>0
\end{aligned}
$$

if $u_{0} \neq 0$. Next, we show that $A_{\varepsilon}$ and $A_{0}$ are compact operators. Let $\left\{f_{n}\right\}$ be a bounded sequence in $L^{2}\left(\Omega_{\varepsilon}\right)$. Then, estimate (19) implies that there exists a constant $c$ such that

$$
\left\|A_{\varepsilon} f_{n}\right\|_{L^{2}\left(\Omega_{\varepsilon}\right)}=\left\|u_{\varepsilon, n}\right\|_{L^{2}\left(\Omega_{\varepsilon}\right)} \leq\left\|u_{\varepsilon, n}\right\|_{H^{1}\left(\Omega_{\varepsilon}\right)} \leq k_{1}\left\|f_{n}\right\|_{L^{2}\left(\Omega_{\varepsilon}\right)} \leq c .
$$

Hence, there exist a subsequence of $\left\{u_{\varepsilon, n}\right\}$ and $\tilde{u}_{\varepsilon} \in H^{1}\left(\Omega_{\varepsilon}\right)$ such that $u_{\varepsilon, n_{k}} \rightarrow \tilde{u}_{\varepsilon}$ weakly in $H^{1}\left(\Omega_{\varepsilon}\right)$ and thus strongly in $L^{2}\left(\Omega_{\varepsilon}\right)$ which exactly means that $A_{\varepsilon}$ is compact. Moreover, (19) implies that $\left\|A_{\varepsilon} f\right\|_{L^{2}\left(\Omega_{\varepsilon}\right)} \leq k_{1}\|f\|_{L^{2}\left(\Omega_{\varepsilon}\right)}$ for any $f \in L^{2}\left(\Omega_{\varepsilon}\right)$. Hence, $\sup _{\varepsilon}\left\|A_{\varepsilon}\right\|_{L\left(H_{\varepsilon}\right)} \leq k_{1}$. The compactness of $A_{0}$ can be proved analogously by applying estimate (20) instead of (19).

Let us verify the condition C3 is fulfilled. Take $f \in L^{2}(\Omega)$. It follows by (18) that

$$
\left\|A_{\varepsilon} R_{\varepsilon} f-R_{\varepsilon} A_{0} f\right\|_{L^{2}\left(\Omega_{\varepsilon}\right)}=\left\|A_{\varepsilon} f-A_{0} f\right\|_{L^{2}\left(\Omega_{\varepsilon}\right)}=\left\|u_{\varepsilon}-u_{0}\right\|_{L^{2}(\Omega)} \rightarrow 0
$$

as $\varepsilon \rightarrow 0$.

Let us verify that the condition $C 4$ is satisfied. Consider a sequence $\left\{f_{\varepsilon}\right\}$, where $f_{\varepsilon} \in$ $L^{2}\left(\Omega_{\varepsilon}\right)$ such that $\sup _{\varepsilon}\left\|f_{\varepsilon}\right\|_{L^{2}\left(\Omega_{\varepsilon}\right)}<+\infty$. Then,

$$
\left\|A_{\varepsilon} f_{\varepsilon}\right\|_{H^{-1}\left(\Omega_{\varepsilon}\right)}=\left\|u_{\varepsilon}\right\|_{H^{-1}(\Omega)} \leq k_{1}\left\|f_{\varepsilon}\right\|_{L^{2}\left(\Omega_{\varepsilon}\right)}<+\infty,
$$

due to (19). The Rellich imbedding theorem implies that the sequence $\left\{A_{\varepsilon} f_{\varepsilon}\right\}$ is compact in $L^{2}(\Omega)$. Thus, there exists a subsequence $\left\{\varepsilon_{k}\right\}$ and $w_{0} \in L^{2}(\Omega)$ such that

$$
A_{\varepsilon_{k}} f_{\varepsilon_{k}} \rightarrow w^{0} \quad \text { as } \varepsilon_{k} \rightarrow 0 .
$$

From this, we deduce that $\left\|A_{\varepsilon_{k}} f_{\varepsilon_{k}}-R_{\varepsilon_{k}} w_{0}\right\|_{L^{2}\left(\Omega_{\varepsilon_{k}}\right)} \rightarrow 0$ as $\varepsilon_{k} \rightarrow 0$. Hence, all the conditions $\mathrm{C} 1-\mathrm{C} 4$ are valid.

Let $\lambda_{\varepsilon}$ be an eigenvalue of the $-\Delta$ operator with the boundary conditions given in (16) and $\nu_{\varepsilon}$ the corresponding eigenvector. In this notation, we have that $-\Delta v_{\varepsilon}=\lambda_{\varepsilon} \nu_{\varepsilon}$ and thus $A_{\varepsilon}\left(\lambda_{\varepsilon} v_{\varepsilon}\right)=v_{\varepsilon}$. From this, it is evident that $A_{\varepsilon} v_{\varepsilon}=\left(1 / \lambda_{\varepsilon}\right) v_{\varepsilon}$. From this, it follows that $\mu_{\varepsilon}^{k}=1 / \lambda_{\varepsilon}^{k}$ ( $\mu_{\varepsilon}^{k}$ is defined in (10)). In the same way, we can deduce that $\mu_{0}^{k}=1 / \lambda_{0}^{k}$. Using the estimate (12), we have

$$
\left|\frac{1}{\lambda_{\varepsilon}^{k}}-\frac{1}{\lambda_{0}^{k}}\right| \leq \frac{1}{1-\lambda_{0}^{k} \beta_{\varepsilon}^{k}} \sup _{v \in N\left(\mu_{0}^{k}, A_{0}\right),\|v\|_{L^{2}(\Omega)}=1}\left\|A_{\varepsilon} R_{\varepsilon} v-R_{\varepsilon} A_{0} v\right\|_{L^{2}\left(\Omega_{\varepsilon}\right)} .
$$

Recall that $N\left(\mu_{0}^{k}, A_{0}\right)=\left\{v \in H_{0}: A_{0} v=\mu_{0}^{k} v\right\}$. Let $v \in N\left(\mu_{0}^{k}, A_{0}\right)$. If we choose $f=v$ in the problem (17), then the solution, denoted by $u_{0}^{v}$, can be expressed as 


$$
u_{0}^{v}=A_{0} v=\mu_{0}^{k} v .
$$

Similarly, if we choose $f=R_{\varepsilon} v$ in the problem (16), then the solution, denoted by $u_{\varepsilon}^{v}$, is of the form

$$
u_{\varepsilon}^{v}=A_{\varepsilon} R_{\varepsilon} v .
$$

In this notation, (29) reads

$$
\left|\frac{1}{\lambda_{\varepsilon}^{k}}-\frac{1}{\lambda_{0}^{k}}\right| \leq \frac{1}{1-\lambda_{0}^{k} \beta_{\varepsilon}^{k}} \sup _{v \in N\left(\mu_{0}^{k}, A_{0}\right),\|v\|_{L^{2}(\Omega)}=1}\left\|u_{\varepsilon}^{v}-R_{\varepsilon} u_{0}^{v}\right\|_{L^{2}\left(\Omega_{e}\right)} .
$$

In [6], it was proved that (17) is the limit problem corresponding to (16). By the results in [6], it follows that there exists a constant $C_{1}$ such that

$$
\left\|u_{\varepsilon}-u_{0}\right\|_{L^{2}(\Omega)} \leq C_{1}\left(\|f\|_{L^{\infty}(\Omega)}\left(\sqrt{\eta_{\mu}}+\left|\frac{\eta_{\mu}}{\varepsilon}-p\right|\right)+\left\|u_{0}\right\|_{L^{\infty}(\Omega) \sqrt{\varepsilon \eta_{\mu}}}\right)
$$

for any $f \in L^{\infty}(\Omega)$. In particular, for the present choice of $f, f=v$, we have

$$
\left\|u_{\varepsilon}^{v}-u_{0}^{v}\right\|_{L^{2}(\Omega)} \leq C_{1}\left(\|v\|_{L^{\infty}(\Omega)}\left(\sqrt{\eta_{\mu}}+\left|\frac{\eta_{\mu}}{\varepsilon}-p\right|\right)+\mu_{0}^{k}\|v\|_{L^{\infty}(\Omega)} \sqrt{\varepsilon \eta_{\mu}}\right) .
$$

This together with the fact that eigenfunctions belong to $C^{\infty}(\bar{\Omega})$ gives that there is a constant $C_{2}$ (which depends on $k$ ) such that

$$
\sup _{v \in N\left(\lambda_{0}^{k}, A_{0}\right),\|v\|_{L^{2}(\Omega)}=1}\left\|u_{\varepsilon}^{v}-u_{0}^{v}\right\|_{L^{2}\left(\Omega_{\varepsilon}\right)} \leq C_{2}\left(\sqrt{\eta_{\mu}}+\left|\frac{\eta_{\mu}}{\varepsilon}-p\right|+\sqrt{\varepsilon \eta_{\mu}}\right) .
$$

From this and (30), we obtain

$$
\left|\frac{1}{\lambda_{\varepsilon}^{k}}-\frac{1}{\lambda_{0}^{k}}\right| \leq \frac{1}{1-\lambda_{0}^{k} \beta_{\varepsilon}^{k}} C_{2}\left(\sqrt{\eta_{\mu}}+\left|\frac{\eta_{\mu}}{\varepsilon}-p\right|+\sqrt{\varepsilon \eta_{\mu}}\right) .
$$

By Lemma 3, we have that $1-\lambda_{0}^{k} \beta_{\varepsilon}^{k}>0$ for sufficiently small values of $\varepsilon$ (as $\beta_{\varepsilon}^{k} \rightarrow 0$ ). Hence, there exists a constant $C$, independent of $\varepsilon$, such that

$$
\left|\frac{1}{\lambda_{\varepsilon}^{k}}-\frac{1}{\lambda_{0}^{k}}\right| \leq C\left(\sqrt{\eta_{\mu}}+\left|\frac{\eta_{\mu}}{\varepsilon}-p\right|+\sqrt{\varepsilon \eta_{\mu}}\right)
$$

and the proof is complete.

As a consequence of the proof above we have the following result:

Corollary 5 The eigenvalues $\lambda_{\varepsilon}^{k}$ of (2) converge to the corresponding eigenvalue $\lambda_{0}^{k}$ of (3).

Proof. We note that by (33)

$$
\left|\lambda_{0}^{k}-\lambda_{\varepsilon}^{k}\right|=\lambda_{\varepsilon}^{k} \lambda_{0}^{k}\left|\frac{1}{\lambda_{\varepsilon}^{k}}-\frac{1}{\lambda_{0}^{k}}\right| \leq \lambda_{\varepsilon}^{k} \lambda_{0}^{k} C\left(\sqrt{\eta_{\mu}}+\left|\frac{\eta_{\mu}}{\varepsilon}-p\right|+\sqrt{\varepsilon \eta_{\mu}}\right) .
$$

It follows from (33) that $\left\{\lambda_{\varepsilon}^{k}\right\}$ is bounded. Hence,

$$
\left|\lambda_{0}^{k}-\lambda_{\varepsilon}^{k}\right| \leq \lambda_{\varepsilon}^{k} \lambda_{0}^{k} C\left(\sqrt{\eta_{\mu}}+\left|\frac{\eta_{\mu}}{\varepsilon}-p\right|+\sqrt{\varepsilon \eta_{\mu}}\right) \rightarrow 0
$$

as $\varepsilon \rightarrow 0$. 
Remark 6 The result established in Theorem 4 is valid for a wide class of domains with perforation along the boundary. It is possible to estimate the difference $\left|K_{\varepsilon}-K_{0}\right|$ more precisely in some partial cases. For some particular cases, it is in fact possible to construct an asymptotic expansion for $K_{\varepsilon}$ with respect to $\varepsilon$ as $\varepsilon \rightarrow 0$ via the method of matching of asymptotic expansion (see, e.g., [11]).

We end this work by giving an example where the result in Theorem 4 is applicable.

Example 7 Consider a semi-strip $B=\left\{\xi \in \mathbb{R}^{2}: 0<\xi_{1}<1, \xi_{2}>0\right\}$ and a bounded domain $T \subset B$ with smooth boundary $\partial T$. Let $T_{\mu}=\left\{\xi \in B: \mu^{-1}\left(\xi-\xi_{0}\right) \in \bar{T}, \xi_{0} \in B\right\}, \mu \in(0,1]$. For this case, the following estimate was derived in [6] for sufficiently small $\mu<\mu_{0}, \mu_{0} \in(0,1]$ :

$$
\frac{2 \pi}{(1+\varepsilon) \ln \mu(\varepsilon)} \leq \eta_{\mu(\varepsilon)} \leq \frac{2 \pi}{\ln \mu_{0}-\ln \mu(\varepsilon)} .
$$

Let us choose $\mu(\varepsilon)=e^{-A / \varepsilon}$, where $A>0$. Then, $\ln \mu(\varepsilon)=-A / \varepsilon$. In this case, the estimate (34) leads to

$$
\frac{2 \pi}{(1+\varepsilon) A} \leq \frac{\eta_{\mu(\varepsilon)}}{\varepsilon} \leq \frac{2 \pi}{\varepsilon \ln \mu_{0}+A}
$$

Hence, $\eta_{\mu(\varepsilon)} / \varepsilon \rightarrow 2 \pi / A=p$. By (15) and (35), we have that

$$
\begin{aligned}
\left|K_{\varepsilon}-K_{0}\right| & \leq C\left(\sqrt{\frac{2 \pi \varepsilon}{\varepsilon \ln \mu_{0}+A}}+\sqrt{\frac{2 \pi \varepsilon^{2}}{\varepsilon \ln \mu_{0}+A}}\right) \\
& +C \max \left\{\frac{2 \pi}{\varepsilon \ln \mu_{0}+A}-\frac{2 \pi}{A}, \frac{2 \pi}{A}-\frac{2 \pi}{(1+\varepsilon) A}\right\} .
\end{aligned}
$$

It can be observed that if $\mu(\varepsilon)=\varepsilon^{\alpha}, \alpha>0$, then (34) gives that $\eta_{\mu(\varepsilon)} / \varepsilon \rightarrow \infty$. This means that the perforation is vanishing too slow in order to have Robin boundary conditions in the limit problems (3) and (17).

Remark 8 The result obtained in this article can be generalized to higher dimensional domain. The crucial step is to prove the estimate similar to (31). This is a good future research problem.

\section{Acknowledgements}

The authors thank Professor Gregory A. Chechkin for valuable remarks. The article was completed during the stay of Yulia Koroleva as Post Doc at Luleå University of Technology in 2010-2011 and was supported by the Luleå University of Technology (Sweden) and partially by the RFBR (Project 12-01-00214). The study of the third author was supported by a grant from the Swedish Research Council (Project 621-2008-5186). We also thank both referees for several suggestions, which have improved the final version of this article.

\section{Author details}

'Department of Differential Equations, Faculty of Mechanics and Mathematics, Moscow Lomonosov State University, Moscow 119991, Russia ${ }^{2}$ Department of Engineering Sciences and Mathematics, Luleå University of Technology, SE97187 Luleå, Sweden ${ }^{3}$ Narvik University College, Postboks 385, 8505 Narvik, Norway

\section{Authors' contributions}

All authors carried out the proofs. All authors conceived of the study, and participated in its design and coordination. All authors read and approved the final manuscript.

\section{Competing interests}

The authors declare that they have no competing interests. 


\section{References}

1. Chechkin, GA, Gadyl'shin, RR, Koroleva, YuO: On the eigenvalue of the Laplace operator in a domain perforated along the boundary. Dokl Acad Nauk. 81(3), 337-341 (2010)

2. Chechkin, GA, Gadyl'shin, RR, Koroleva, YuO: On the asymptotic behavior of a simple eigenvalue of a boundary-value problem in a domain perforated along the boundary. Diff Equ. 47(6), 822-832 (2011). doi:10.1134/50012266111060073

3. Chechkin, GA, Koroleva, YuO, Persson, LE: On the precise asymptotics of the constant in the Friedrich's inequality for functions, vanishing on the part of the boundary with microinhomogeneous structure. J Inequal Appl 2007, 13 (2007). Article ID 34138

4. Chechkin, GA, Koroleva, YuO, Meidell, A, Persson, LE: On the Friedrichs inequality in a domain perforated nonperiodically along the boundary. Homogenization procedure. Asymptotics in parabolic problems. Russ J Math Phys. 16(1), 1-16 (2009). doi, 10.1134/S1061920809010014

5. Koroleva, YuO: On the Friedrich's-type inequality in a three-dimensional domain aperiodically perforated along a part of the boundary. Russ Math Surv. 65(4), 199-200 (2010)

6. Belyaev, AG: On singular perturbations of boundary-value problems. PhD Thesis. Moscow State University, Moscow (1990) (in Russian)

7. Belyaev, AG: Homogenization of a Fourier boundary-value problem for Poisson equation in a domain perforated along the boundary. Russ Math Surv. 45(4), 123 (1990)

8. Sobolev, SL: Some applications of functional analysis in mathematical physics. Translations of Mathematical Monographs, 90. American Mathematical Society, Providence (1991)

9. Oleinik, OA, Shamaev, AS, Yosifian, GA: Mathematical Problems in Elasticity and Homogenization. North-Holland, Amsterdam (1992)

10. Oleinik, OA, Shamaev, AS, Yosifian, GA: On a limit behavior of spectrum of the sequence of operators, which are defined in different Hilbert spaces. Russ Math Surv. 44(3), 157-158 (1989)

11. Il'in, AM: Matching of asymptotic expansions of solutions of boundary value problems. Translations of Mathematical Monographs, 102. American Mathematical Society, Providence (1992)

doi:10.1186/1029-242X-2011-129

Cite this article as: Koroleva et al:: On Friedrichs-type inequalities in domains rarely perforated along the boundary. Journal of Inequalities and Applications 2011 2011:129.

\section{Submit your manuscript to a SpringerOpen ${ }^{\odot}$ journal and benefit from:}

- Convenient online submission

- Rigorous peer review

- Immediate publication on acceptance

- Open access: articles freely available online

- High visibility within the field

- Retaining the copyright to your article

Submit your next manuscript at $\boldsymbol{\wedge}$ springeropen.com 